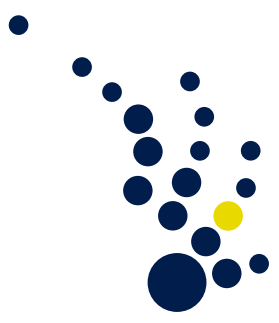

Institut für Mumerische Simulation

Rheinische Friedrich-Wilhelms-Universität Bonn

Endenicher Allee 19b 553115 Bonn • Germany phone +49228 73-69828

www.ins.uni-bonn.de

\author{
M. Griebel, M. Klitz
}

\title{
Simulation of micron-scale drop impact
}

INS Preprint No. 1811

October 2018 



\title{
Simulation of micron-scale drop impact
}

\author{
M. Griebel ${ }^{\mathrm{a}}$, M. Klitz ${ }^{\mathrm{a}, \mathrm{b}, *}$ \\ ${ }^{a}$ Institute for Numerical Simulation, University of Bonn, Wegelerstr. 6, 53115 Bonn \&3 \\ Fraunhofer Institute for Algorithms and Scientific Computing (SCAI), Schloss \\ Birlinghoven, 53754 Sankt Augustin. \\ ${ }^{b}$ German Aerospace Center (DLR), Simulation and Software Technology, Linder Höhe, \\ 51147 Cologne, Germany.
}

\begin{abstract}
The numerical simulation of droplet impact is of interest for a vast variety of industrial processes, where practical experiments are costly and timeconsuming. In these simulations, the dynamic contact angle is a key parameter, but the modeling of its behavior is poorly understood so far. One of the few models which considers the overall physical context of the involved 'moving contact line problem' is Shikhmurzaev's interface formation model [1]. In addition to keeping the problem well-posed, all surface and bulk parameters, such as the contact angle, are determined as part of the solution rather than being prescribed functions of contact line speed. In this article, we couple an asymptotic version of the interface formation model with our three-dimensional incompressible two-phase Navier-Stokes solver. Additionally, we employ a simple constant contact angle approach. We compare the results of these two numerical models with those from laboratory experiments for the micron-scale droplet impact on substrates with a variety of wetting characteristics. With our sophisticated asymptotic interface formation model, the droplet shapes, heights and diameters compare very well with practical experiments.
\end{abstract}

Keywords: micron-scale drop impact, Navier-Stokes, dynamic contact angle, interface formation model, CLSVOF

\section{Introduction}

The numerical simulation of droplets impacting and spreading on solid surfaces is of critical importance for a number of industrial applications such as spray painting or coating and ink-jet printing as well as the delivery of

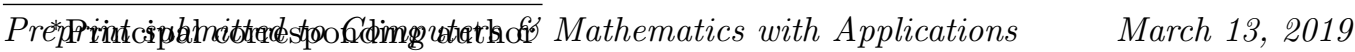

Email addresses: griebel@ins.uni-bonn.de (M. Griebel), margrit.klitz@dlr.de (M. Klitz) 

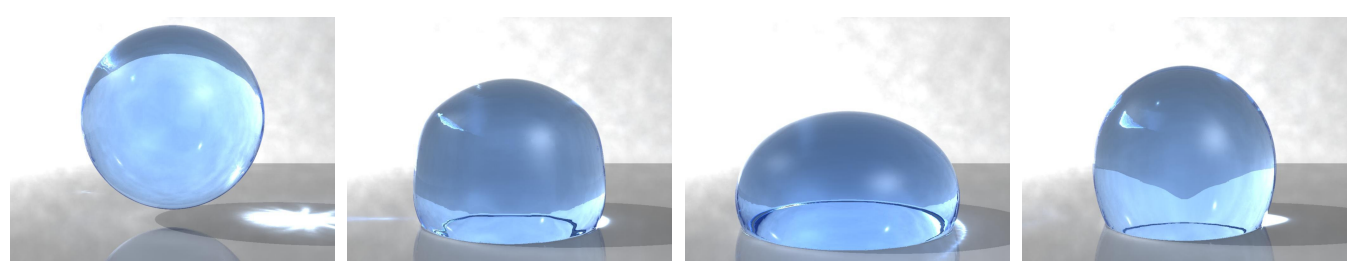

Figure 1: Simulation of dynamic wetting with a water droplet.

15

agricultural chemicals and the coating of tablets in the pharmaceutical industry [2]. Spray painting, for example, is a technique where a distribution of droplets is sprayed through the air onto a surface. Thereby, protective coatings as well as decorative finishes are applied to manufactured products. An important step to understand the overall process on a microscopic level is to study a single droplet's impact onto a surface. This physical process itself already exhibits a wide range of interesting phenomena. A simulation example of a water droplet impacting on a solid substrate is shown in Figure 1.

Most experimental studies of drop impaction focus on drops in a millimeterscale regime. However, in ink-jet printing and other industrial processes such as spray coating or drop spraying, the drop size ranges from sub-microns to a several hundred microns. In order to study the applicability of results on the millimeter-scale to micron-scale drops, practical experiments were conducted in [3], where the impact of micron-scale water droplets on surfaces with several different equilibrium contact angles was compared to the impact of millimeter droplets consisting of a mixture of water and glycerin. Moreover, Lattice Boltzmann simulations of these droplets can be found in [4], where the contact angle is reconstructed by the empirical 'Hoffman's function' and the capillary number.

A key parameter for the simulation of dynamic wetting and in particular of droplet impact is the contact angle $\theta$, which is measured through the liquid at the contact line and quantifies the wettability of the substrate. Due to the contact line motion, $\theta$ becomes dynamic as well and can change considerably from its well-understood static value. So far, The existing theoretical and numerical approaches often fail to correctly predict the results of practical experiments - mostly due to the moving contact line problem. One of the few models, which considers the overall physical context of such processes is Shikhmurzaev's interface formation model [1]. In addition to keeping the problem well-posed, all surface and bulk parameters, such as the contact angle, are determined as part of the solution rather than being prescribed 
functions of contact line speed. Furthermore, this model is able to describe a large variety of other flows with singularities including coalescence, break-up and formation of cusps.

The interface formation model is currently under debate from both its physical and its numerical point of view. Physical challenges mainly concern a missing condition at the contact line. This was first pointed out in [5] and later on confirmed in [6] with the argument that a further closure relation is required to describe the dissipative process of mass transfer from one interface across the contact line to another. Numerical challenges are provided by the complexity of the full interface formation model. So far, it has only been implemented in a two-dimensional finite element framework [7] to study axisymmetric drop impact [8] and coalescence [9]. Moreover, in the interface formation model, different length scales are involved that need to be matched to the non-dimensional similarity parameters. In [7], practical recommendations on the spatial resolution are provided to capture the physics associated with the smallest length scale. Although parts of these estimates might be transferable to the different flow regimes in three dimensions, the overall complexity of the interface formation model remains: In three dimensions, we would have to solve the Navier-Stokes equations for the bulk flow along with the interface formation equations at the free surface and liquid-solid interface. These equations are themselves partial differential equations and have to be complemented with boundary conditions at the contact line. To our knowledge, there is no implementation in three dimensions yet. Due to these reasons, we deal with a reduction of the full interface formation model at small capillary numbers $\mathrm{Ca}$. Then, we do not have to solve equations on the interface itself, and the scaling as well as the coupling with the nonlinear Navier-Stokes equations become much more straightforward to handle.

The asymptotic version of Shikhmurzaev's interface formation model has been scarcely studied so far $[10,11,12]$. Only recently, it has been integrated into a three-dimensional immersed boundary-based framework for dynamic contact angles [13]. Similar to our approach, the asymptotic approach serves as a boundary condition of the Continuum Surface Force method and fares well for droplets of millimeter size. In this work, we focus on micron-scale droplets instead.

The contribution of this article is as follows: We couple an asymptotic version of Shikhmurzaev's interface formation model with our three-dimensional incompressible two-phase Navier-Stokes solver NaSt3DGPF [14, 15]. With this sophisticated model, we study micron-scale drop impact. The computed 
droplet shapes and the heights and widths of the droplets are compared to practical experiments from [3]. Furthermore, we give a qualitative comparison of our results with those obtained by the Lattice Boltzmann simulation in $[4]$.

The remainder of this article is organized as follows: In Section 2, we discuss the mathematical details of our approach. We describe the Navier-Stokes equations and the interface capturing coupled level-set and volume-of-fluid method (CLSVOF). We then explain how the contact angle can be included as a boundary condition for the level-set function and describe the mathematical details of the contact angle model by Shikhmurzaev [1]. In Section 3, we address our numerical model. Here, we describe the discretization of our two-phase Navier-Stokes solver NaSt3DGPF in space and time. Additionally, we focus on the implementation of the contact angle boundary condition and of the reduced interface formation model. In Section 4, we present and discuss our numerical experiments. We use the asymptotic interface formation model (AIFM) by Shikhmurzaev and a simple constant contact angle approach (CCA) to study the micron-scale drop impact of four drops with impact velocities $4.36 \mathrm{~m} \mathrm{~s}^{-1}$ and $12.2 \mathrm{~m} \mathrm{~s}^{-1}$ on two different substrates with equilibrium contact angles $31^{\circ}$ and $107^{\circ}$. The heights and widths of the droplets are compared to those observed in practical experiments conducted in [3] and to the numerical results in [4]. We conclude this article by a discussion and suggestions for further research.

\section{The mathematical model}

The simulation of droplet impact requires the consideration of two immiscible fluids and the treatment of the free surface in between. A popular method for implicitly capturing free surface motion is the level-set (LS) method introduced in [16], where a smooth scalar field $\phi$ is advected with the flow, and the zero level-set of this field represents the interface $\Gamma_{f}$. Then, $\phi>0$ in the liquid and $\phi<0$ in the gas. In our setting, surface tension effects are included via the Continuum Surface Force (CSF) method [17].

On the whole domain $\Omega$ the one-fluid continuum formulation of the twophase Navier-Stokes equations can be written as

$$
\begin{aligned}
\rho(\phi)\left(\partial_{t} \boldsymbol{u}+(\boldsymbol{u} \cdot \nabla \boldsymbol{u})\right)+\nabla p & =\nabla \cdot(\mu(\phi) \boldsymbol{S})-\sigma \kappa(\phi) \delta(\phi) \nabla \phi+\rho(\phi) \boldsymbol{g} \\
\nabla \cdot \boldsymbol{u} & =0 \\
\phi_{t}+\boldsymbol{u} \cdot \nabla \phi & =0
\end{aligned}
$$


with time $t \in[0, T]$, fluid velocity $\boldsymbol{u}=(u, v, w)^{\top}$, pressure $p$, stress tensor $\boldsymbol{S}=\nabla \boldsymbol{u}+(\nabla \boldsymbol{u})^{\top}$, surface tension $\sigma$ and a volume force $\boldsymbol{g}$. In this equation, $\kappa=\nabla \cdot \boldsymbol{n}$ is the curvature with the outward normal $\boldsymbol{n}=\nabla \phi /|\nabla \phi|$, where ${ }_{115}$ $|\cdot|$ denotes the Euclidean norm and $\phi$ is the level-set function. Furthermore, $\mu(\phi)$ is the viscosity and $\rho(\phi)$ the density, and they are both defined in dependence of $\phi$ as in [18]. The Dirac delta functional is denoted by $\delta(\phi)$ as used in [17]; see also [18, 19, 20] for details and for appropriate boundary conditions for the velocity, pressure and level-set function.

Furthermore, we complement our LS function $\phi$ by the volume-of-fluid (VOF) function $F$ which results in the Coupled Level-Set Volume-Of-Fluid (CLSVOF) method [21]. Like $\phi$, the function $F$ tracks the interface and is transported by

$$
F_{t}+\boldsymbol{u} \cdot \nabla F=0
$$

Then, both $\phi$ and $F$ contribute to a geometrical reconstruction of the interface: The smooth $\phi$ is used to compute the surface normal while the massconservative $F$ is used to correct the mass enclosed by the zero level-set of $\phi$.

Within the level-set method the interface between the two fluids is given by the zero level-set of $\phi$ as $\Gamma_{f}(t)=\{\boldsymbol{x}: \phi(\boldsymbol{x}, t)=0\}$ for all times $t \in[0, T]$ and $\boldsymbol{x} \in \mathbb{R}^{3}$, and the continuous $\phi$ is advected by the pure transport equation (1). For the CLSVOF method, we have to transport the discontinuous VOF function $F$. To this end, let $W \subset \Omega$ be an arbitrary small fluid volume. For $\phi>0$ for the liquid phase and $\phi<0$ for the gas phase, we define $F$ as

$$
F(W):=\frac{1}{|W|} \int_{W} H(\phi(x, y, z)) d x d y d z
$$

with the Heaviside function

$$
H(\phi):= \begin{cases}0 & \text { if } \phi<0 \\ \frac{1}{2} & \text { if } \phi=0 \\ 1 & \text { if } \phi>0\end{cases}
$$

Within this integral formulation, the transport equation (2) becomes welldefined in a weak sense. With partial integration we obtain

$$
\frac{\partial}{\partial t} \int_{W} H(\phi(x, y, z)) d \boldsymbol{x}+\int_{\partial W} H(\phi(x, y, z)) \boldsymbol{u} \cdot \boldsymbol{n} d s=0
$$

where $\boldsymbol{n}$ denotes the outward normal on $\partial W$. Thus, the change of liquid ${ }_{125}$ volume contained in $W$ equals the volume flux across the boundary of $W$, 


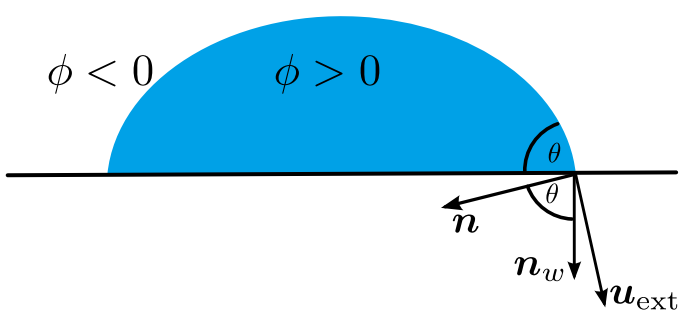

Figure 2: Boundary conditions for $\phi . n$ is normal to the interface and points from lower to higher level set values and $\boldsymbol{n}_{w}$ is the outward normal drawn from the active flow region into the geometry region. $\boldsymbol{u}_{\text {ext }}$ is the extension velocity of a related approach described in [22].

which formally describes volume conservation and is the basis of all VOF methods [19]. After discretization, we employ (3) in each grid cell, so that $F$ becomes the discontinuous liquid volume fraction on the whole domain; cf. Section 3 for our discretization strategy. Then, $F=1$ in a cell full of liquid, $F=0$ in a cell full of gas and $0<F<1$ in cells which contain the interface. We refer to [21] for all details on the implementation of the CLSVOF method.

\subsection{Contact angle boundary condition for the level-set method}

For the transport equation in (1), boundary conditions for the LS and the VOF function are required, which determine the shape of the free surface at the contact line and depend on the dynamic contact angle $\theta_{d}$ as soon as the contact line is moving.

We follow $[20,23]$ and formulate a Neumann boundary condition for the level-set function, which incorporates the dynamic contact angle. Thus, at the boundary of $\Omega$ the contact angle is defined by the geometric relation $\boldsymbol{n} \cdot \boldsymbol{n}_{w}=\cos (\theta)$, where $\theta$ is the contact angle (static or dynamic) and $\boldsymbol{n}_{w}$ is the outward normal drawn from the flow region into the boundary; see Figure 2. Then, at any wall of $\Omega$, whose outward normal is given by $\boldsymbol{n}_{w}^{i}= \pm \boldsymbol{e}^{i}$ for $i \in\{1,2,3\}$, the level-set's $i$-th derivative $\phi_{x_{i}}$ can be related to $\theta$ by

$$
\phi_{x_{i}}= \pm \cot (\theta) \sqrt{\sum_{j=1, j \neq i}^{3} \phi_{x_{j}}^{2}}
$$

for any angle $0<\theta<\pi$; see [20].

Note that our approach can be considered as a special case of the extension velocity approach [22], where the contact angle is taken into account by extrapolating the liquid interface, represented by the level-set method, into the solid. This requires the construction of an appropriate extension velocity $\boldsymbol{u}_{\text {ext }}$ which is also shown in Figure (2). For further details on this approach and the connection to ours, see [20, 22]. 


\subsection{Modeling the Contact Angle}

In this subsection we present a model based on Shikhmurzaev's interface formation theory [1]. The full model accounts for different classes of flows, where interfaces are formed or destroyed. The equations, which capture the surface tension relaxation process and have to be solved on the surface itself, are derived from mass, momentum and energy conservation. For the case of small capillary and Reynolds numbers, we can analyze them as a local problem whose solution can be incorporated into various types of global flow problems.

The full analytical interface formation model is quite complicated and is described in detail in [1]. For numerical realizations a reduced model is recommended. For such a reduction of the full interface formation model at small capillary numbers $C a$, we follow the description in [1]. There, the flow domain is split into two asymptotic regions, and in both regions the limit $\mathrm{Ca} \rightarrow 0$ is studied analytically. In the inner asymptotic region to leading order in $\mathrm{Ca}$, the dynamic contact angle and the dimensionless contact-line speed $V$ are related by

$$
\cos \left(\theta_{e}\right)-\cos \left(\theta_{d}\right)=\frac{2 V\left[\cos \left(\theta_{e}\right)-\tilde{\sigma}_{s g}+\left(1-\rho_{G}^{s}\right)^{-1}\left(1+\rho_{G}^{s} u_{(12)}\left(\theta_{d}, k_{\mu}\right)\right)\right]}{V+\left[V^{2}+1+\left(\cos \left(\theta_{e}\right)-\tilde{\sigma}_{s g}\right)\left(1-\rho_{G}^{s}\right)\right]^{\frac{1}{2}}}
$$

with $\theta_{e}$ the equilibrium contact angle, $k_{\mu}$ the gas-to-liquid viscosity ratio, and $\rho_{G}^{s}=\rho_{e}^{s} / \rho_{(0)}^{s}$ the dimensionless surface density. Here, $\rho_{e}^{s}$ is the equilib- ${ }^{155}$ rium surface density and $\rho_{(0)}^{s}$ is a phenomenological constant describing the surface density for zero surface tension. Furthermore, $\tilde{\sigma}_{s g}=\sigma_{s g} / \sigma$ is the dimensionless surface tension in the gas-solid interface obtained by division with the equilibrium liquid-gas surface tension $\sigma$.

The dimensionless contact line speed in equation (7) is given by

$$
V=u_{\mathrm{cl}} \sqrt{\frac{\tau \beta}{\gamma \rho_{0}^{s}(1+4 \alpha \beta)}} .
$$

We introduce the scaling factor $S c$ by

$$
S c=\sqrt{\frac{\sigma^{2} \tau \beta}{\mu^{2} \gamma \rho_{0}^{s}(1+4 \alpha \beta)}},
$$

and obtain therefore

$$
V=\frac{u_{\mathrm{cl}} \mu}{\sigma} S c
$$


Here, $\sigma$ is the equilibrium surface tension, $u_{\mathrm{cl}}$ is the contact line velocity, $\alpha$ and $\beta$ are phenomenological constants depending on the 'state of the interface', $\gamma$ is a phenomenological constant describing the compressibility of the fluid, and $\tau$ is the surface tension relaxation time which can be treated as a material constant. Thus, $S c$ depends on the material properties of the fluid and the interface.

The radial velocity $u_{(12)}\left(\theta_{d}, k_{\mu}\right)$ in equation (7) must be derived from the solution in the outer region.

For our approach, we replace $u_{(12)}\left(\theta_{d}, k_{\mu}\right)$ by the inner limit of the outer solution as described in [1], i.e. by a numerically computed far field velocity sufficiently close to the contact line. This alters the dynamic contact angle for the same contact line speed and is exactly what is observed in laboratory experiments as the non-local influence of the flow field/geometry on the dynamic contact angle, i.e. the hydrodynamic assist of wetting.

An alternative approach is to derive $u_{(12)}\left(\theta_{d}, k_{\mu}\right)$ from the solution in the outer region. In particular, in the outer region to leading order in $\mathrm{Ca}$ the freesurface curvature becomes zero and we obtain a flow problem in a wedge [1]. The solution to this problem is given by Moffatt in [24] as

$$
u_{(12)}\left(\theta_{d}, 0\right)=\frac{\sin \theta_{d}-\theta_{d} \cos \theta_{d}}{\sin \theta_{d} \cos \theta_{d}-\theta_{d}} .
$$

In the following, we use the abbreviation AIFM to refer to the asymptotic interface formation model (7). Within the AIFM we use both of the above approaches for the computation of the radial velocity. If the standard one fails to provide a solution, we use the alternative (10) instead. For the purpose of comparison we will also use a constant contact angle approach (CCA) later on, where the contact angle is fixed to its equilibrium value at all times, i.e. $\theta_{d}=\theta_{e}$.

\section{The Numerical Model}

For the discretization of the two-phase Navier-Stokes equations (1) in time we employ time stepping $t^{n+1}=t^{n}+\Delta t, n=0,1, \ldots$, where the choice of $\Delta t$ ensures the stability of our discretization with the well-known CFLcondition. Due to the explicit treatment of the surface tension $\sigma$, the time step is mainly restricted by

$$
\Delta t<\left[\frac{\left(\rho_{1}+\rho_{2}\right) \cdot h^{2} \cdot \varepsilon}{2 \pi \sigma}\right]^{\frac{1}{2}},
$$


where $\rho_{1,2}$ are the densities in the two fluid phases, $h$ is the minimum grid resolution, and $\varepsilon=1.9 \mathrm{~h}$ is the interface thickness in the CSF-method [17].

Specifically, we use an explicit second-order Adams-Bashforth time integration scheme. The solution process is based on the well-known projection method: First, an intermediate velocity field $\boldsymbol{u}^{*}$, which may not be divergence free, is advanced by the Adams-Bashforth time scheme; second, we compute a correction $\nabla p^{n+1}$ of the intermediate velocity field by the pressure Poisson equation which leads to a divergence free velocity field $\boldsymbol{u}^{n+1}$. Thus, we treat the pressure implicitly and solve the Poisson equation by a Jacobi-preconditioned conjugate gradient method. In order to track the interface we employ the CLSVOF method [21] where we transport both $\phi$ and $F$ by second-order operator splitting in time.

In space, we employ a Cartesian staggered grid with grid cells

$$
\left[x_{i-1 / 2}, x_{i+1 / 2}\right] \times\left[y_{j-1 / 2}, y_{j+1 / 2}\right] \times\left[z_{k-1 / 2}, z_{k+1 / 2}\right],
$$

and we define the discrete computational domain $\Omega_{i, j, k}$ as a union of these cells. For $\{i, j, k\} \in \mathbb{Z}$ we use the notation $\delta x_{i}=x_{i+1 / 2}-x_{i-1 / 2}$, with analogous definitions of $\delta y_{j}$ and $\delta z_{k}$. Furthermore, 'ghost cells' or 'boundary cells' are needed in up to three additional strips of cells attached to $\Omega_{i, j, k}$, which are necessary for the discretization of large finite difference stencils and boundary conditions.

On $\Omega_{i, j, k}$ we employ the finite difference method: The diffusion term 200 is computed by second-order central differences. A fifth-order weighted essentially non-oscillatory (WENO) scheme is used for the discretization of the convective terms of the Navier-Stokes equations. Surface tension is evaluated using a smoothed delta function and third order interpolation. In addition, the CLSVOF method requires the geometric reconstruction of the interface, 205 where we opt for the piecewise linear interface calculation so that the interface is approximated by straight planes perpendicular to the surface normal vector of the interface in each cell. To this end, we compute a piecewise linear reconstructed LS function $\phi^{R}$ in such a way that it is as close as possible to the real LS function $\phi$ while $F$ ensure mass conservation. Note that we reinitialize the LS function by the exact signed normal distance of each cell center to the reconstructed interface: The reconstructed interface consists of piecewise linear plane segments as well as the plane segments at the cell boundaries that connect a plane in one cell with the plane in the cell next to it. The magnitude of $\phi$ is calculated as the shortest distance from the cell ${ }_{215}$ center to any of the reconstructed interface segments. 
The parallelization of the code is based on conventional domain decomposition techniques using Message Passing Interface (MPI). The discretization and the solver are described in more detail in [25, 18]. For the CLSVOF and related methods see also $[20,21]$ and the references cited therein.

\subsection{Discretization of the contact angle boundary condition}

The discretization of the contact angle boundary condition (6) is very similar to the discretization of the standard Neumann boundary condition for the LS function. We exemplify this in the $y$-direction, where equation (6) becomes

$$
\phi_{y}=-\cot (\theta) \sqrt{\phi_{x}^{2}+\phi_{z}^{2}}
$$

On the staggered grid, the LS values $\phi$ are discretized at the cell center. Then, with grid cells denoted by integers $(i, j, k)$,

$$
\frac{\phi_{i, j, k}-\phi_{i, j-1, k}}{\delta y_{j}}=-\cot (\theta) \sqrt{\phi_{x_{i, j, k}}^{2}+\phi_{z_{i, j, k}}^{2}},
$$

where the values of $\phi_{i, j, k}=\phi_{i, j, k}(t)$ are evaluated at the same time step $t=t^{n}$, and $\delta y_{j}$ is the mesh width in $y$-direction. The derivatives $\phi_{x_{i, j, k}}$ and $\phi_{z_{i, j, k}}$ can be discretized by central differences. The wall $y=0$ lies in between the cell $(i, j=1, k)$ and the ghost cell $(i, j=0, k)$. At this wall, we can solve equation (14) with $j=1$ for the staggered grid's ghost cell value $\phi_{i, 0, k}$, which gives the required boundary condition for $\phi$.

The values for the contact angle $\theta$ are computed by the discretized dynamic contact angle model by Shikhmurzaev (7), which we will discuss in the following.

\subsection{Discretization of the asymptotic interface formation model}

In this section, we describe the incorporation of the AIFM (7) as as well as of the constant contact angle approach (CCA) into our two-phase NavierStokes solver.

In its time-discrete formulation the model (7) is given by

$$
\begin{gathered}
\cos \left(\theta_{e}\right)-\cos \left(\theta_{d}^{n+1}\right)= \\
\frac{2 V^{n+1}\left[\cos \left(\theta_{e}\right)-\tilde{\sigma}_{s g}+\left(1-\rho_{G}^{s}\right)^{-1}\left(1+\rho_{G}^{s} u_{(12)}^{n+1}\left(\theta_{d}^{n+1}, k_{\mu}\right)\right)\right]}{V^{n+1}+\left[\left(V^{n+1}\right)^{2}+1+\left(\cos \left(\theta_{e}\right)-\tilde{\sigma}_{s g}\right)\left(1-\rho_{G}^{s}\right)\right]^{\frac{1}{2}}}
\end{gathered}
$$


with $\theta_{e}$ the equilibrium contact angle, $k_{\mu}$ the gas-to-liquid viscosity ratio, and $\rho_{G}^{s}$ and $\tilde{\sigma}_{s g}$ phenomenological material constants; compare Subsection 2.2. Here, $n+1$ denotes the time step $t^{n+1}=t^{n}+\Delta t, n=0,1, \ldots$ The dimensionless contact line speed in equation (15) is given by

$$
V^{n+1}=\frac{u_{\mathrm{cl}}^{n+1} \mu}{\sigma} S c .
$$

with the scaling factor $S c$ described by equation $(8) ; \mu$ is the viscosity and $\sigma$ the equilibrium surface tension. Here, the contact line velocity $u_{\mathrm{cl}}^{n+1}$ is determined by the motion of the contact line position, i.e.

$$
u_{\mathrm{cl}}^{n+1}=\frac{x_{p}^{n+1}-x_{p}^{n}}{\delta t},
$$

Note that we apply our contact angle model only on a single point and not ${ }^{235}$ on the whole contact line. This means that we assume that the drop spreads symmetrically, although our simulation itself is fully three-dimensional. The assumption of symmetry underlies many droplet impacts, but for a wider range of applications, the dynamic contact angle will have to be computed at each point along our contact line - not at a single reference point only.

In order to solve (15), we require the computation of the contact line velocity $u_{\mathrm{cl}}^{n+1}$ as well as the computation of the radial velocity $u_{(12)}^{n+1}$.

First, however, we have to find an approximation for the position $x_{p}$ of the contact line for a given discrete point in time $t=t^{n}$ (for now we omit the superscript $n$ ). We exemplify this computation on the domain boundary $y=0$ (cf. Fig. 3). Then, the contact angle $\theta_{d}$, the contact point $x_{p}$ and the contact line velocity $u_{\mathrm{cl}}$ need to be evaluated at one grid point only. Let $i_{\max }$ and $k_{\max }$ denote the maximum number of grid cells in $x$ - and $z$-direction. Now, we compute the contact point $x_{p}$ as follows: For $i=i_{\max } / 2, \ldots, i_{\max }$, we check the values of $\phi_{i, 1, k_{\max } / 2}$ along this line. As soon as $\phi$ changes its sign, we store the values $\phi_{\text {in }}=\phi_{i=\text { in }, 1, k_{\max } / 2}$ and $\phi_{\text {out }}=\phi_{i=\text { out }, 1, k_{\max } / 2}$ of the LS function inside and outside the droplet along with the absolute position of the cell centers $x_{\mathrm{in}}=x_{i=\mathrm{in}, 1, k_{\max } / 2}$ and $x_{\mathrm{out}}=x_{i=\mathrm{out}, 1, k_{\max } / 2}$. We approximate the position of the contact point by the weighted average

$$
x_{p}=\frac{\left|\phi_{\mathrm{in}}\right| x_{\mathrm{out}}+\left|\phi_{\mathrm{out}}\right| x_{\mathrm{in}}}{\left|\phi_{\mathrm{in}}\right|+\left|\phi_{\text {out }}\right|},
$$

so that the position of the cell center nearer to the contact line contributes more to the computation of $x_{p}$ than the one further away. 


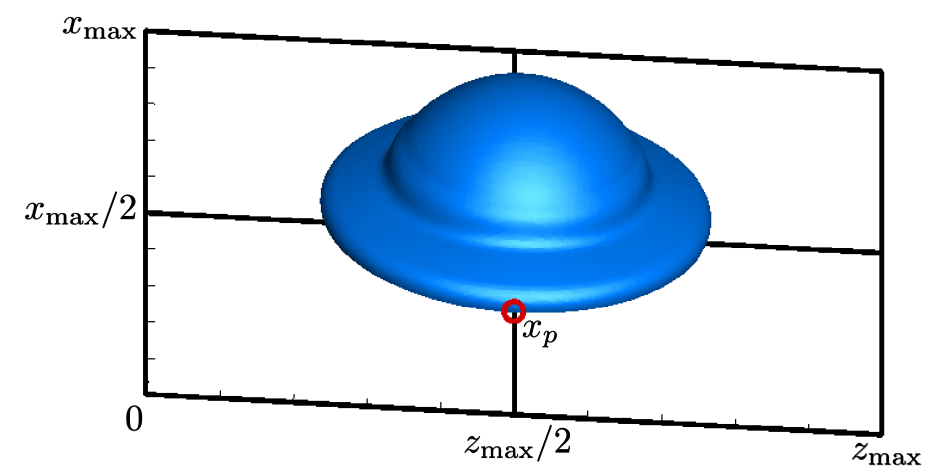

Figure 3: The contact line velocity is evaluated at the contact point $x_{p}$ at the intersection with the line $z=z_{\max } / 2$.

245 In our approach AIFM, we replace $u_{(12)}^{n+1}$ by the inner limit of the outer solution, i.e. by a numerically computed far field velocity sufficiently close to the contact line. This far field velocity value is arbitrarily chosen to be about two grid cells away from the contact line. To this end, we set $u_{(12)}^{n+1}=u_{\mathrm{in}-1,2, k_{\max } / 2}^{n+1}$. We can now solve (15) directly by evaluating the arccosfunction.

The alternative is to compute the radial velocity $u_{(12)}^{n+1}$ as the solution to a flow problem in a wedge (10). We discretize this equation as

$$
u_{(12)}^{n+1}\left(\theta_{d}^{n+1}, 0\right)=\frac{\sin \theta_{d}^{n+1}-\theta_{d}^{n+1} \cos \theta_{d}^{n+1}}{\sin \theta_{d}^{n+1} \cos \theta_{d}^{n+1}-\theta_{d}^{n+1}} .
$$

Then, the contact angle equation (15) becomes nonlinear and we have to invoke a Newton iteration method to solve for $\theta_{d}^{n+1}$.

To summarize, the contact line model AIFM as well as the constant contact angle approach (CCA) fit into our flow solver as follows:

1. Let $\theta^{n}$ and the contact line position $x_{p}^{n}$ be given from the previous time step.

2. Solve the LS advection equation in (1) in conjunction with the contact angle boundary condition (14) and $\theta^{n}$.

3. Determine $x_{p}^{n+1}$ from (17) and use equation (16) for $u_{\mathrm{cl}}^{n+1}$. 
4. Depending on the contact angle approach

- CCA: Set $\theta^{n+1}=\theta_{e}$.

- AIFM: Use the far field velocity value $u_{(12)}^{n+1}=u_{\mathrm{in}-1,2, k_{\max } / 2}^{n}$ and solve (15) directly for $\theta^{n+1}$. If the argument of the arccos is not in $[-1,1]$, we use Moffatt's solution (18) and invoke a Newton iteration method to solve (15) for $\theta^{n+1}$.

5. Perform the reinitialization of $\phi$ in the CLSVOF method with the contact angle boundary condition (14) and the new $\theta=\theta^{n+1}$.

Note that we use the no-slip condition for the velocity for both contact angle models CCA and AIFM. On the staggered grid, the no-slip condition is never exactly fulfilled, which introduces enough numerical slip to eliminate the stress singularity at the contact line.

Let us stress the potential problems of our implementation of the contact angle models: The use of the no-slip condition, as well as our choice of the radial velocity value near the contact line for AIFM, make our numerical solution undesirably dependent on the choice of the underlying grid: For a 275 very fine grid, we have less slip and $u_{(12)}$ is evaluated closer to the contact line than on a coarser grid. An alternative would be the use of a slip or Navierslip condition. Furthermore, $u_{(12)}$ should have a fixed distance to the contact line. However, the choice remains problematic, since this value should be both sufficiently near the contact line and a part of the far field solution. Therefore, the position of $u_{(12)}$ will at least remain a problem-dependent parameter and cannot be finally determined. In this article, we keep the grid fixed but as soon as grid refinement is studied, both the boundary condition and $u_{(12)}$ should be adapted accordingly.

\section{Numerical Results}

We now use AIFM and CCA to study the micron-scale drop impact of four drops with impact velocities $4.36 \mathrm{~m} \mathrm{~s}^{-1}$ and $12.2 \mathrm{~m} \mathrm{~s}^{-1}$ on two different substrates with equilibrium contact angles $31^{\circ}$ and $107^{\circ}$. In all simulations, the interface is tracked by the CLSVOF method. The heights and widths of the droplets are compared to those observed in practical experiments conducted 290 in [3]. The numerical results with our sophisticated AIFM offer a substantial improvement over the much simpler CCA.

Our parallel simulations are computed on the Atacama cluster of the Institute for Numerical Simulation [26], Bonn University. This cluster consists 


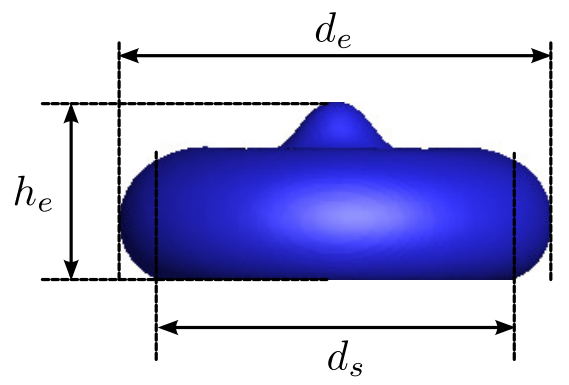

Figure 4: Illustration of the measurement of the droplet's height and diameter. In the practical experiment, $d_{e}$ is measured near the contact line and $h_{e}$ is the droplet's maximum height. In the simulation, $d_{s}$ is measured directly at the contact line and can slightly differ from $d_{e}$.

of 78 Dell PowerEdge M620 nodes with 1264 cores and has a Linpack performance of $20630 \mathrm{GFlop} / \mathrm{s}$, while maintaining a total memory of $4992 \mathrm{~GB}$.

\subsection{Postprocessing}

In the version of the fluid solver NaSt3DGPF that we use here, the state of the simulation is saved in one binary data file for each computational time step that is required for analysis. These binary files are converted to a visualization file format. We then use two different software tools for post processing, namely ParaView [27] and Tecplot 360 (Release 1, 2013) [28]. Both are able to extract the iso 0 level-set surface. We use Tecplot for visualization purposes only, while we use ParaView also for the computation of the droplet height and width over time. ParaView relies on the Marching Cubes algorithm [29] for isosurface extraction. For details on the applied filters; see [20].

Our post processing could still be handled on a desktop computer in reasonable time. If we move towards finer meshes, memory requirement as well as disk space will become an issue. Then, we could still use ParaView in parallel or employ the version of NaSt3DGPF described in [30], where an isosurface extraction method is implemented directly into the fluid solver.

Let us explain how we measure the droplet's diameter and height for the comparison with practical experiments. In the practical experiment, the droplet height $h_{e}$ is defined as its maximum height, and the diameter $d_{e}$ is its maximum width measured macroscopically 'near' the substrate. In our simulation, the droplet diameter $d_{s}$ is measured directly at the contact line, which can differ slightly from the measurement of $d_{e}$ as illustrated in 
Table 1: Impact velocity, diameter and dimensionless numbers for the impact of water.

\begin{tabular}{ccccccc}
\hline Liquid & $v_{0} / \mathrm{m} \mathrm{s}^{-1}$ & $d_{0} / 10^{-6} \mathrm{~m}$ & $R e$ & $W e$ & $O h$ & $\theta_{e}$ \\
\hline distilled water & 4.36 & 48.8 & 238 & 12.8 & 0.0151 & $31^{\circ}$ and $107^{\circ}$ \\
distilled water & 12.2 & 50.5 & 689 & 103 & 0.0148 & $31^{\circ}$ and $107^{\circ}$ \\
\hline
\end{tabular}

Figure 4. Furthermore, the simulation droplet height $h_{s}$ is always measured in the middle of the droplet, which is not always possible in experiments, 320 e.g., when the outer rim is higher than its middle part.

To account for these differences we conduct a post-processing step with the ParaView software, in which we measure the outline of the droplet over time ${ }^{1}$, whereby we obtain the diameter $d_{p}$ and the height $h_{p}$, which both correspond to the actual maximum extension in the respective directions. 325 Then, we define the final simulation height as $h:=\max \left(h_{s}, h_{p}\right)$, so that our run-time results are corrected if the highest point of the droplet is not its center. Furthermore, we choose the final simulation diameter as $d=d_{p}$ if $d_{p} \geq d_{s}$ and if $d_{p}$ is measured near the substrate (when the droplet rebounds, its maximum extension is found far from the substrate). Thereby, the final 330 simulation droplet height $h$ and diameter $d$ are measured as similar to the practical experiment as possible.

\subsection{Units and dimensionless quantities}

In our computations we are not using dimensionless quantities, and for the micron-scale numerical experiments, we convert all input parameters from 335 meters to millimeters. For example, the density $\rho=1000 \mathrm{~kg} \mathrm{~m}^{-3}$ is converted to $\rho=1000 \cdot 10^{-9} \mathrm{~kg} \mathrm{~mm}{ }^{-3}$. This conversion is necessary due to the computational accuracy: When we use meters instead of millimeters, the LS function, which is a distance function, can no longer be accurately defined since the involved distances simply become too small.

The involved dimensionless numbers are summarized in Table 1. These are the Ohnesorge number $O h=\frac{\mu_{l}}{\rho_{l} \sigma_{l g} d_{0}}$, and the Weber number $W e=$

\footnotetext{
${ }^{1}$ We here use ParaView to show the zero contour of the level-set function. Then, in the contour's 'information' menu, the maximum $x$-, $y$ - and $z$-ranges of the droplet can be found.
} 
Table 2: Material parameters for distilled water.

\begin{tabular}{cccc}
\hline & $\rho / \mathrm{kg} \mathrm{m}^{-3}$ & $\mu / 10^{-3} \mathrm{~kg} \mathrm{~m}^{-1} \mathrm{~s}^{-1}$ & $\sigma_{l g} / 10^{-2} \mathrm{~N} \mathrm{~m}^{-1}$ \\
\hline \multicolumn{4}{c}{ distilled water (micron-scale impact) } \\
\hline liquid & 1000 & 8.93 & 7.2 \\
air & 1.184 & 0.01826 & \\
\hline \hline
\end{tabular}

$\frac{\rho_{l} v_{0}^{2} d_{0}}{\sigma_{l g}}$. Here, $\mu_{l}$ is the liquid viscosity, $\rho_{l}$ is the liquid density, $\sigma_{l g}$ is the liquidgas interfacial tension, $v_{0}$ is the impact speed and $d_{0}$ is the initial droplet

345

diameter. An alternative to the definition of the Ohnesorge number is the use of the Reynolds number $R e=\frac{\sqrt{W e}}{O h}$. The Weber number is a measure of the fluid's inertia compared to its surface tension, the Ohnesorge number relates the viscous forces to inertial and surface tension forces, and the Reynolds number quantifies the relative importance of inertial and viscous forces.

\subsection{Definition of the numerical experiment}

We consider a domain $\Omega$ filled with air and a droplet of water impacting on two substrates with equilibrium contact angles $31^{\circ}$ and $107^{\circ}$, respectively. The first substrate is a thermally oxidized silicon wafer, while the second substrate consists of three self-assembled monolayers on a gold-coated silicon wafer. In the practical experiment, the wafer is prepared in such a way that the amounts of hydrophilic $(\mathrm{OH})$ and hydrophobic $\left(\mathrm{CH}_{3}\right)$ groups vary in the monolayers; see [3]. Here, we consider the case of $100 \% \mathrm{CH}_{3}$ in the three selfassembled monolayers only. In the following, we refer to this substrate as S107 and to the thermally oxidized silicon wafer as S31 due to their equilibrium contact angles of $107^{\circ}$ and $31^{\circ}$ with water.

On these two substrates, we use the approaches AIFM and CCA to study the micron-scale drop impact with two impact velocities $4.36 \mathrm{~m} \mathrm{~s}^{-1}$ and $12.2 \mathrm{~m} \mathrm{~s}^{-1}$. We summarize the relevant physical and material parameters in Tables 1 and 2. All in all, we perform eight different simulations within our numerical experiment, whose distinctive features are summarized in Table 3.

For our simulations, we solve the Navier-Stokes equations combined with the CLSVOF method for the capturing of the interface. The numerical interface thickness is $\varepsilon=1.9 h$, where $h \approx 5 \cdot 10^{-7} \mathrm{~m}$ is the fixed equidistant mesh width, and we use a narrow band of four cells for the reinitialization of the 
Table 3: Description of the eight different simulations that we perform in this numerical experiment.

\begin{tabular}{ccccccc}
\hline Experiment & CA model & Liquid & $v_{0} / \mathrm{m} \mathrm{s}^{-1}$ & $d_{0} / 10^{-6} \mathrm{~m}$ & substrate & $\theta_{e}$ \\
\hline 1.0 & AIFM & distilled water & 4.36 & 48.8 & $\mathrm{~S} 31$ & $31^{\circ}$ \\
1.1 & CCA & & & & & \\
2.0 & AIFM & distilled water & 4.36 & 48.8 & $\mathrm{~S} 107$ & $107^{\circ}$ \\
2.1 & CCA & & & & & \\
3.0 & AIFM & distilled water & 12.2 & 50.5 & $\mathrm{~S} 31$ & $31^{\circ}$ \\
3.1 & CCA & & & & & \\
4.0 & AIFM & distilled water & 12.2 & 50.5 & $\mathrm{~S} 107$ & $107^{\circ}$ \\
4.1 & CCA & & & & &
\end{tabular}

Table 4: Parameters for distilled water impacting on S31 with equilibrium contact angle $31^{\circ}$ and on S107 with equilibrium contact angle $107^{\circ}$.

\begin{tabular}{ll}
\hline final time: & $T=50 \mu \mathrm{\mu s}$ \\
flow domain: & $\Omega=(0.15 \times 0.075 \times 0.15) \cdot 10^{-3} \mathrm{~m}^{3}$ \\
material parameters: & see Tables 1 and 2 \\
body force: & $\boldsymbol{g}=(0.0,-9.8,0.0)^{\top} \mathrm{m} \mathrm{s}^{-2}$ \\
\hline interface thickness: & $\epsilon=1.9 h$ \\
grid resolution: & dof $=301 \times 151 \times 301$ \\
grid size: & $h \approx 5 \cdot 10^{-7} \mathrm{~m}$ \\
approximate time step: & $\Delta t \approx 0.02 \mu \mathrm{s}$ \\
employed method: & CLSVOF \\
contact angle model: & AIFM and $\theta_{d}=\theta_{e}$ \\
\hline & Parameters for AIFM \\
dimensionless parameter: & $S c=5.0$ \\
dimensionless surface tension (gas-solid): & $\tilde{\sigma}_{s g}=-0.07$ \\
phenomenological constant: & $\rho_{G}^{s}=0.54$ \\
\hline
\end{tabular}


level-set function. Due to the explicit treatment of surface tension, our time step is mainly restricted by (11). With the simulation parameters in Table 4 , this yields an approximate time step of $\Delta t \approx 0.02 \mu \mathrm{s}$.

In Table 4, we also find the phenomenological constants and the dimensionless parameters of AIFM. These values are taken from [31], where the velocity dependence of the dynamic contact angle is studied experimentally for a series of water-glycerol solutions. Then, the experimental results are compared with those of the interface formation theory. Due to the good agreement between theory and experiments, estimates for the phenomenological parameters $\rho_{G}^{s}$ and $\tilde{\sigma}_{s g}$ can be derived; see [31] and [1, pp. 246-247] for details.

Our simulations are evaluated as follows:

1. We focus on the evolution of the droplet by plotting its zero contour.

2. We compare the droplet shapes that were numerically computed by AIFM and CCA with the ones obtained experimentally by Dong et al. [3].

3. We compute the dimensionless droplet height $H^{*}=h / d_{0}$ and the dimensionless diameter $D^{*}=d / d_{0}$ over time and compare the results of AIFM and CCA to the data from practical experiments provided by [3].

4. We give a qualitative comparison of our results with those obtained by the Lattice Boltzmann simulation in [4].

\subsection{Expected behavior}

All in all, we expect that our results with AIFM are close to the practical experiments for the droplet shapes, the droplet diameter and its height. For all numerical experiments, AIFM should show considerable improvement compared to CCA.

395 Particularly, at early times after impact, when inertial forces are more dominant, we expect good results from both AIFM and CCA. However, from our previous experience with CCA [20], we know that the droplet diameter tends to be overestimated, which can be expected for these numerical experiments as well. Instead, with AIFM, we expect a much better approximation of the experimentally measured diameter.

The Weber number for Exp. 1 and 2 is considerably smaller than for Exp. 3 and 4, i.e. surface tension forces are more dominant in the first two experiments than in the last two. If surface tension effects are dominant, the contact angle model is much more influential. Therefore, we expect larger differences between AIFM and CCA for Exp. 1 and 2. These differences will 
lessen for Exp. 3 and 4. Here, however, we expect AIFM to be very close to the practical experiment, since small errors introduced by the contact angle model have less influence.

\subsection{Discussion of results}

\subsubsection{Experiment 1}

In our first experiment, we use AIFM as well as CCA to simulate the impact of water on S31 with equilibrium contact angle $31^{\circ}, v_{0}=4.36 \mathrm{~m} \mathrm{~s}^{-1}$ and $d_{0}=48.8 \mu \mathrm{m}$ (cf. Tab. 3).

First, we are interested in the droplet shapes computed with AIFM, which are depicted in Figure 5(a). In this case, the deformation of the droplet is rather moderate. At $t=2 \mu \mathrm{s}$, two layers of the droplet develop, which are slightly stretched throughout the simulation during the spreading phase. In the subsequent relaxation phase, these two layers are no longer visible, and the droplet resembles a spherical dome.

In Figure 5(b), we compare the results of the practical experiment (first ${ }_{420}$ row) with simulation results by AIFM (second row) and by CCA, where $\theta_{d}$ is chosen equal to $\theta_{e}$ for the whole simulation time (third row). The droplet shapes in both simulations are very close to those observed in the practical experiment, at least up to about $t=8 \mu \mathrm{s}$. This is to be expected, since initially, surface tension and capillary forces are less dominant, and the droplet shapes depend less on the applied contact angle. However, even in these early times, the diameter of the droplet seems to be overestimated with CCA. This behavior becomes most visible at $t=10$ and $t=30 \mu \mathrm{s}$, where the diameter is considerably too large and the whole droplet is too flat compared to the practical experiment. In contrast, the diameter of the droplet with AIFM is smaller at all times, and the computed droplet shapes are very close to the experimental snapshots.

These findings are confirmed by the computation of the dimensionless diameter $D^{*}=d / d_{0}$ over time in Figure $5(\mathrm{c})$. The diameter in the practical experiment is slightly below the one computed by AIFM from about $t=$ $7 \mu \mathrm{s}$ onwards. In contrast, for CCA, the experimental results are drastically overestimated; much more so than with AIFM for the whole simulation. Most noticeably, from $0 \mu$ s to $7 \mu$ s the diameter computed by AIFM and that of the practical experiment agree almost perfectly, while from about $1 \mu \mathrm{s}$ onwards, the results with CCA already diverge.

In the same Figure 5(c), the dimensionless droplet height $H^{*}=h / d_{0}$ is plotted over time. For the droplet height, the distinction between both 


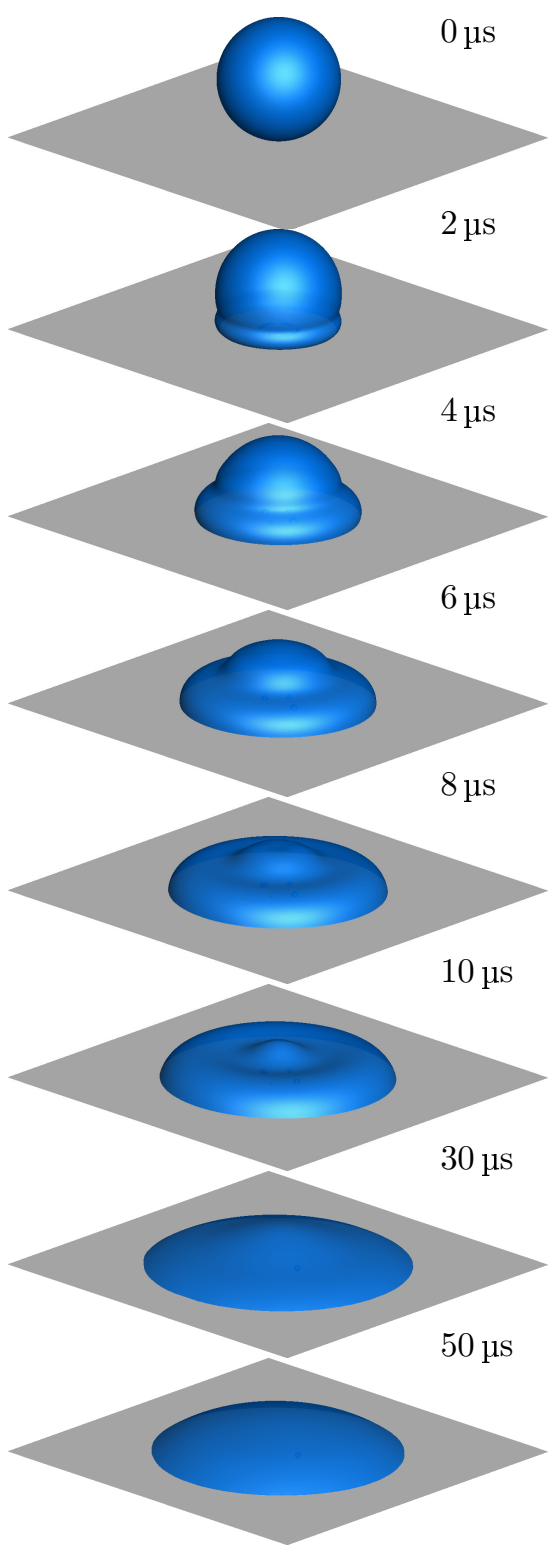

(a) Droplet shapes computed by AIFM for Exp. 1.0 from Table 3.

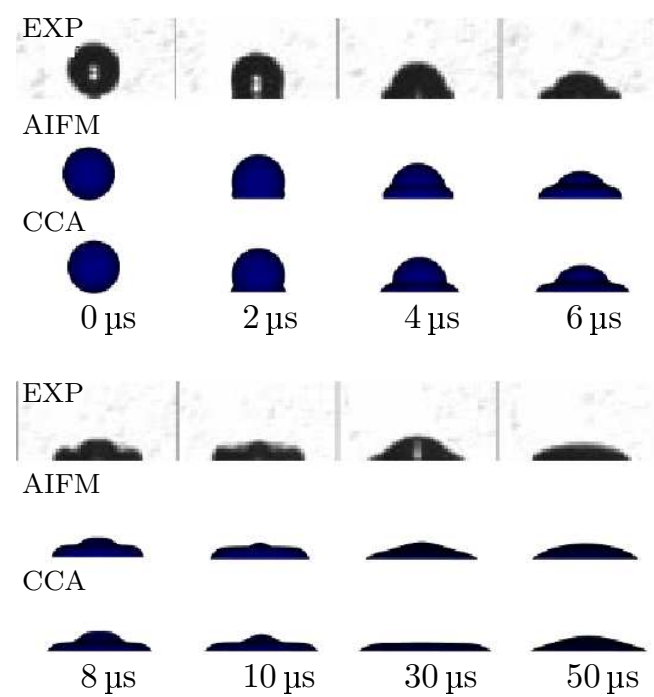

(b) Comparison of the practical experiment (first row), Exp. 1.0 (second row) and Exp. 1.1 (third row); cf. Table 3.

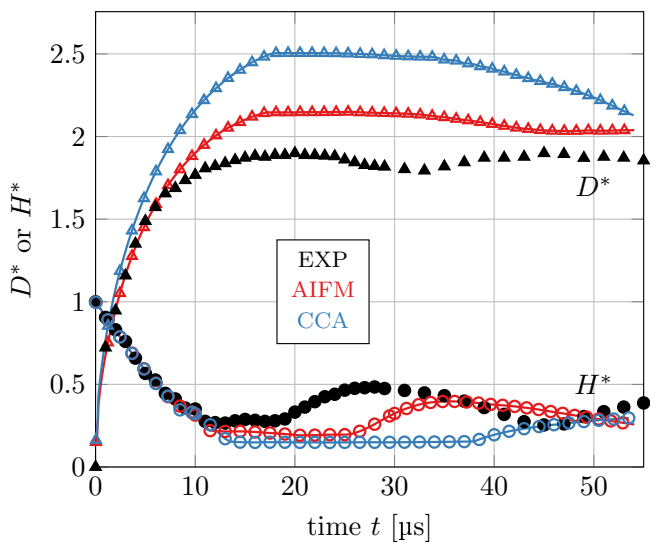

(c) Dimensionless droplet diameter $D^{*}$ (triangles) and height $H^{*}$ (circles) vs. time: EXP (black), AIFM (red) and CCA (blue).

Figure 5: Distilled water impacting on S31 with equilibrium contact angle $31^{\circ}$, impact speed $4.36 \mathrm{~m} \mathrm{~s}^{-1}$, and droplet size $48.8 \mu \mathrm{m}$. Experimental data and figures used with permission by H. Dong [3]. 
contact angle models and the practical experiment becomes less prominent. From $0 \mu$ s to $10 \mu \mathrm{s}$ all results agree perfectly. Then, up to about $t=50 \mu \mathrm{s}$, the droplet height is underestimated with CCA, while AIFM is a little closer to the ${ }_{445}$ experiment. Note that AIFM also predicts the bouncing back of the droplet, but does so about $7 \mu$ s later than it occurs in the practical experiment. Thus, both curves develop in a similar manner, with a shift for the one computed by AIFM. This behavior cannot be observed for CCA, where the curve seems to develop distinctly from the one of the practical experiment.

\subsubsection{Experiment 2}

In our second experiment, we use both models to simulate the impact of the same droplet on another substrate, i.e. on S107 with equilibrium contact angle $107^{\circ}$, which corresponds to Exp. 2 in Table 3.

The droplets' shapes are given in Figure 6(a). Up to about $t=10 \mu \mathrm{s}, \quad{ }^{455}$ the droplet behaves similarly to Exp. 1. Thus, we see the development of two layers from $t=2 \mu \mathrm{s}$ onwards, which are slightly stretched throughout the simulation during the spreading phase. Then, due to the higher contact angle, the droplet recedes in the subsequent relaxation phase, the two layers are no longer visible, and the droplet shape at $t=50 \mu$ s resembles the upper ${ }_{460}$ part of an ellipsoid.

In Figure 6(b), we again compare the results of the practical experiment (first row) with simulation results by AIFM (second row) and those by CCA (third row). Up to $t=6 \mu \mathrm{s}$, the droplet shapes in both simulations are very close to those observed in the practical experiment. However, even in these ${ }_{465}$ early times, the diameter of the droplet seems to be slightly overestimated with CCA. This overestimation increases with time. Furthermore, at $t=$ $10 \mu \mathrm{s}$, a small spherical cap at the droplet's top is still visible for CCA, which is not the case for AIFM and the practical experiment. Then, at $t=30 \mu \mathrm{s}$, the height of the droplet is vastly overestimated while the droplet shape 470 computed by AIFM remains much closer to the experimental snapshot. Before $50 \mu \mathrm{s}$ are reached, the droplet even rebounds for CCA, so that no meaningful numerical results can be obtained after that point in time.

All of these findings are confirmed by the computation of the dimensionless diameter $D^{*}=d / d_{0}$ over time in Figure $6(\mathrm{c})$. The diameter in the ${ }_{475}$ practical experiment is slightly below the one computed by AIFM from about $t=10 \mu \mathrm{s}$ to $47 \mu \mathrm{s}$, after which the behavior reverses. Again, the droplet diameter curve computed by AIFM develops similarly to the one by the practical experiment, but with a slight shift. In particular, up to $10 \mu \mathrm{s}$, the diameter 


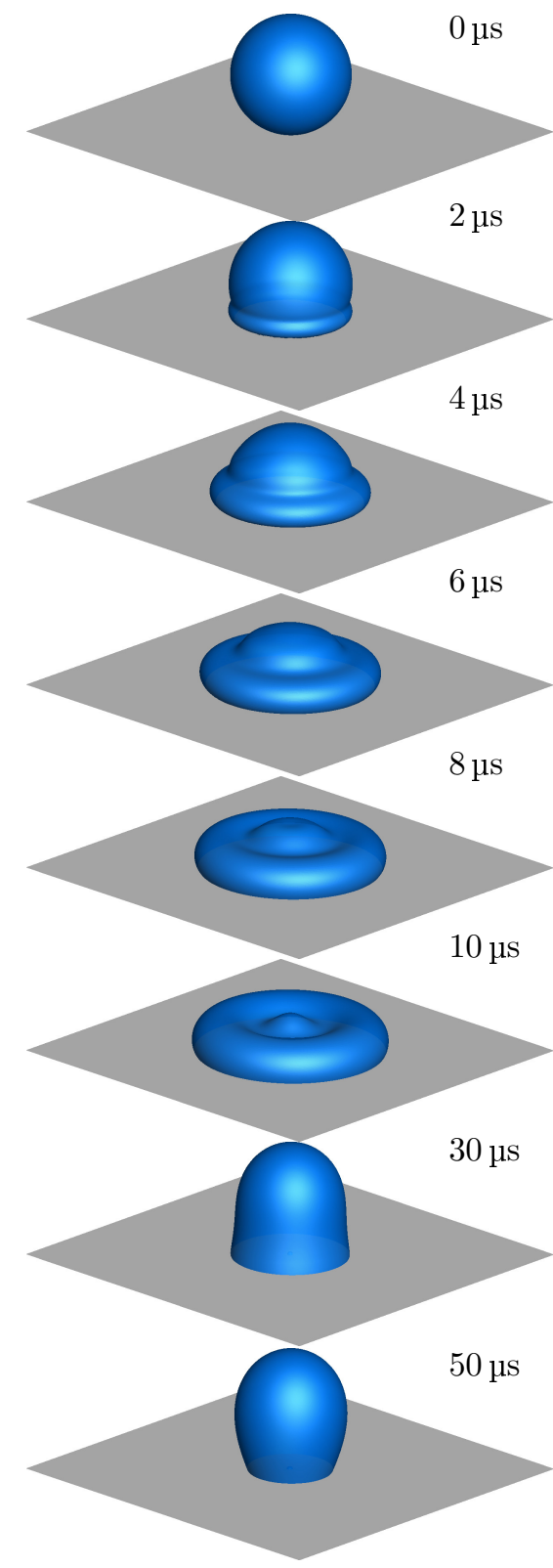

(a) Droplet shapes computed by AIFM for Exp. 2.0 from Table 3.
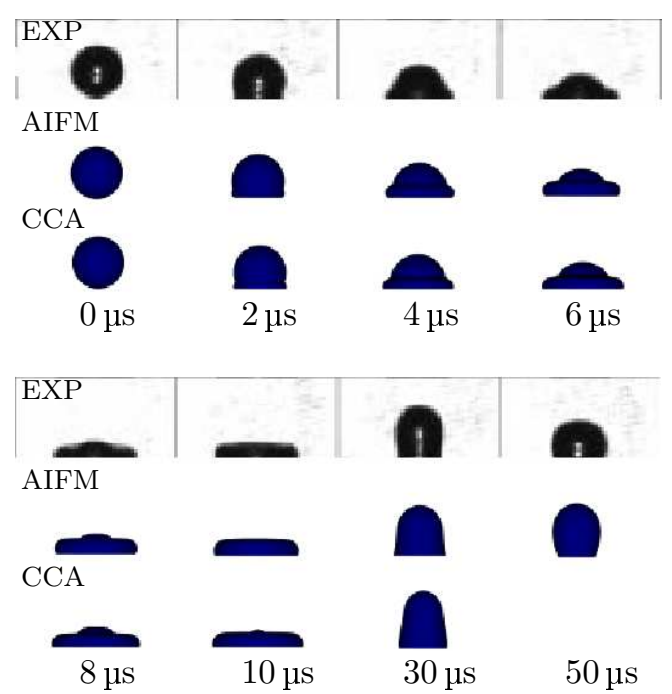

(b) Comparison of the practical experiment (first row), Exp. 2.0 (second row) and Exp. 2.1 (third row); cf. Table 3.

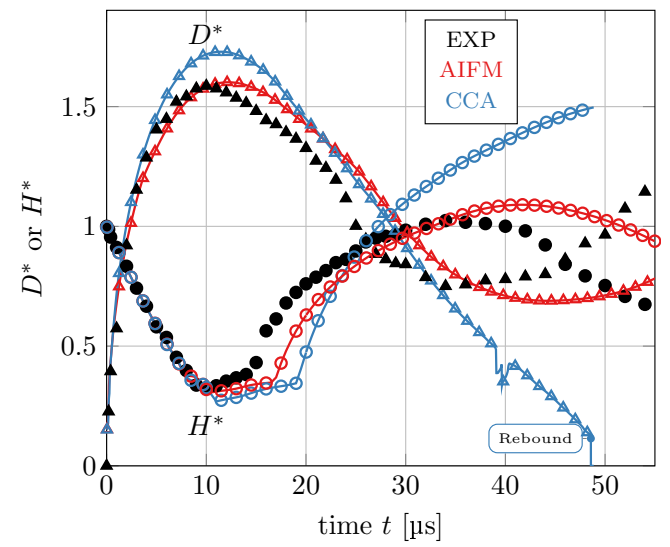

(c) Dimensionless droplet diameter $D^{*}$ (triangles) and height $H^{*}$ (circles) vs. time: EXP (black), AIFM (red) and CCA (blue).

Figure 6: Distilled water impacting on S107 with equilibrium contact angle $107^{\circ}$, impact speed $4.36 \mathrm{~m} \mathrm{~s}^{-1}$, and droplet size $48.8 \mu \mathrm{m}$. Experimental data and figures used with permission by H. Dong [3]. 
is captured perfectly by AIFM. In contrast, for CCA, the experimental results 480 diverge more prominently from the beginning, and the maximum droplet diameter is vastly overestimated. From $t=20 \mu \mathrm{s}$ to $30 \mu \mathrm{s}$, the results are close to AIFM after which the diameter decreases until the rebound of the droplet

In the same Figure 6(c), the dimensionless droplet height $H^{*}=h / d_{0}$ is plotted over time. Again, the droplet height curve computed by AIFM devel- ${ }_{485}$ ops similarly to the one by the practical experiment, and seems to display the same shift as the diameter curve. As in Exp. 1, the distinction between both contact angle models and the practical experiment is less prominent for the droplet height than for the droplet diameter up to about 23 s. In particular, from $0 \mu \mathrm{s}$ to $10 \mu \mathrm{s}$ all results agree almost perfectly. From $t=10 \mu \mathrm{s}$ onwards, 490 the droplet height increases constantly for CCA, until the droplet rebounds. In contrast, AIFM is able to predict the renewed decline of the droplet height, which is earlier on also observed in the practical experiment.

\subsubsection{Experiment 3}

In our third experiment, we use AIFM as well as CCA to simulate the impact of water on S31 with equilibrium contact angle $31^{\circ}, v_{0}=12.2 \mathrm{~m} \mathrm{~s}^{-1}$ and $d_{0}=50.5 \mathrm{\mu m}$ (cf. Tab. 3 ). Thus, we have a slightly larger droplet and an about three times higher initial velocity.

The droplet shapes computed with AIFM are displayed in Figure 7(a). Due to the higher impact speed, the droplet deforms much faster than in the previous examples. At $t=2 \mu \mathrm{s}$, two layers of the droplet develop, which are subsequently stretched. From $t=4 \mu \mathrm{s}$ onwards, the outer rim of the droplet becomes its highest part, while the droplet's middle remains leveled. Finally, at $t=50 \mu$ s the droplet shape resembles a flat spherical cap.

In Figure 7(b), we compare the results of the practical experiment (first 505 row) with simulation results by AIFM (second row) and by CCA (third row). Up to about $t=4 \mu \mathrm{s}$ the droplet shapes in both simulations are quite close to those observed in the practical experiment. However, even in these early times and as already observed previously, the diameter of the droplet seems to be overestimated with CCA, and the difference in diameter between this 510 model and the practical experiment increases with time. Due to this overestimation, at about $t=7 \mu \mathrm{s}$, the droplet touches the walls of our simulation box, so that the results after that point in time can no longer be regarded as reliable. In addition to the too large diameter, the whole droplet is too curved in comparison to the result obtained with AIFM and the experimental 515 snapshot. At all times, in contrast to CCA, the droplet diameter with AIFM 


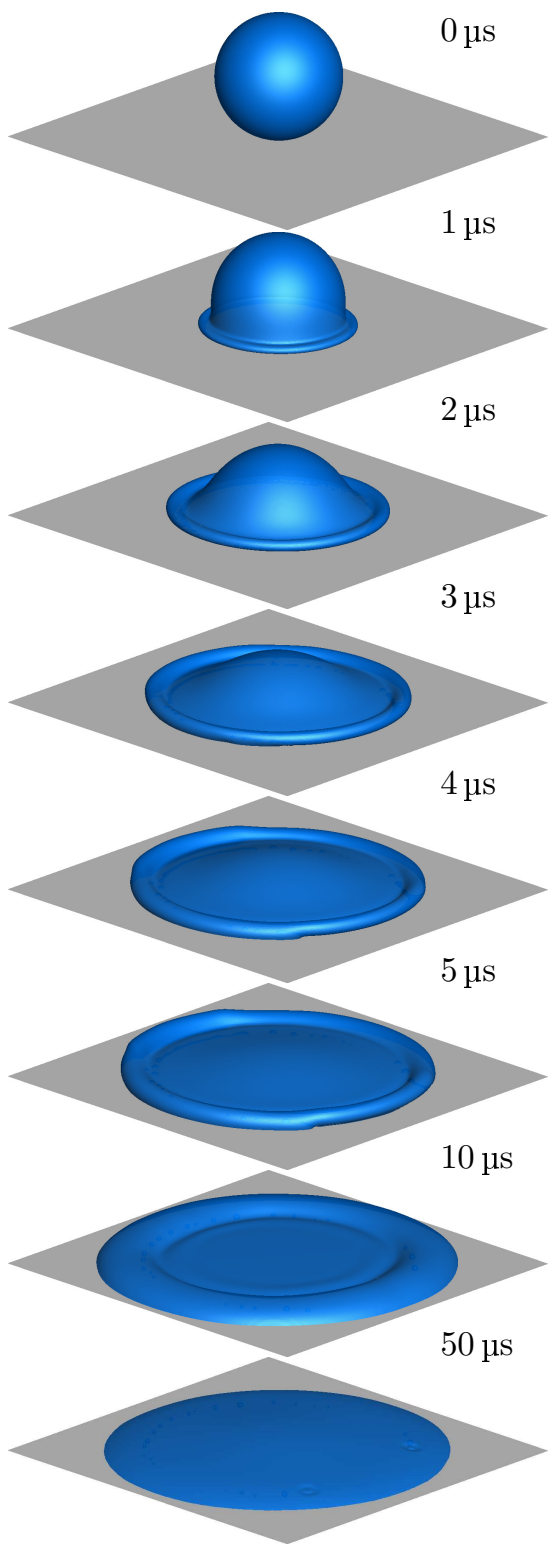

(a) Droplet shapes computed by AIFM for Exp. 3.0 from Table 3.

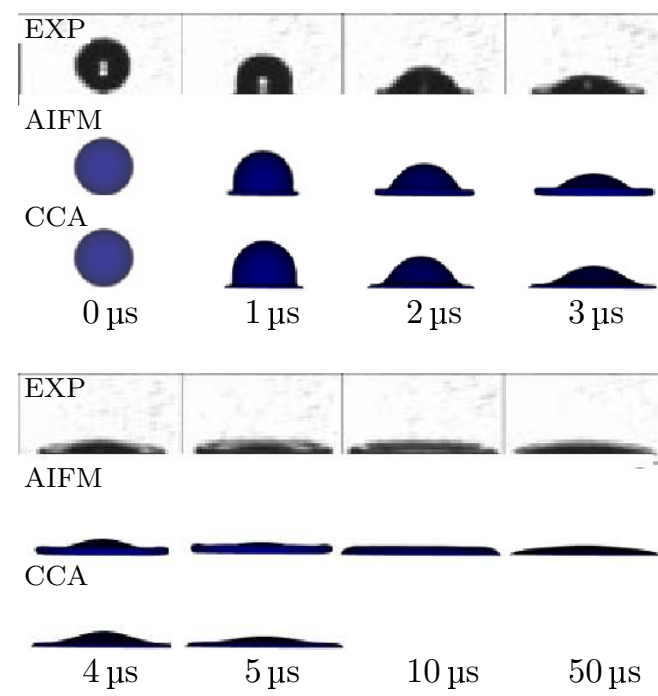

(b) Comparison of the practical experiment (first row), Exp. 3.0 (second row) and Exp. 3.1 (third row); cf. Table 3.

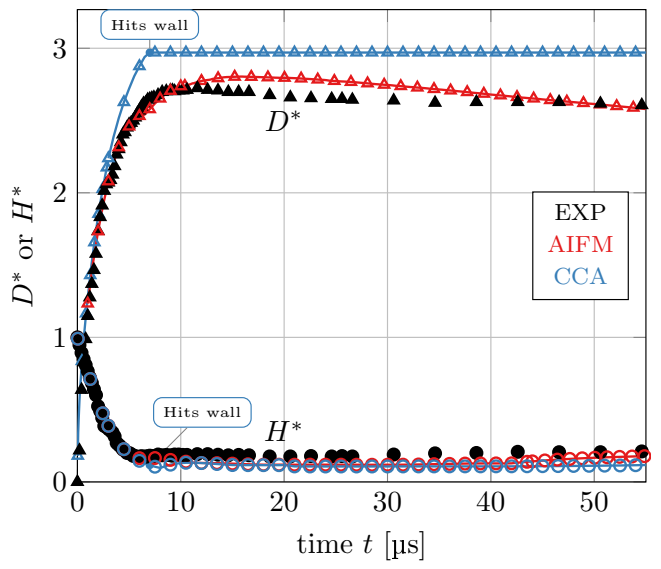

(c) Dimensionless droplet diameter $D^{*}$ (triangles) and height $H^{*}$ (circles) vs. time: EXP (black), AIFM (red) and CCA (blue).

Figure 7: Distilled water impacting on S31 with equilibrium contact angle $31^{\circ}$, impact speed $12.2 \mathrm{~m} \mathrm{~s}^{-1}$, and droplet size $50.5 \mu \mathrm{m}$. Experimental data and figures used with permission by H. Dong [3]. 
is smaller, and the droplet shapes are exceedingly close to the experimental snapshots.

Again, we confirm these qualitative findings by the computation of the dimensionless diameter $D^{*}=d / d_{0}$ over time in Figure $7(\mathrm{c})$. For this ex- ${ }_{520}$ periment, the diameter computed by AIFM and by the practical experiment agree almost perfectly. In contrast, the diameter computed by CCA deviates increasingly from $t=2 \mu \mathrm{s}$ onwards until the walls of the simulation domain are reached.

In the same Figure $7(\mathrm{c})$, the dimensionless droplet height $H^{*}=h / d_{0}$ is ${ }_{525}$ plotted over time. For the droplet height, the distinction between AIFM and CCA is less prominent. Note that the results from about $t=7 \mu$ s onwards are insignificant for CCA, since the droplet touches the walls of our simulation domain. However, from $0 \mu \mathrm{s}$ to $6 \mu \mathrm{s}$ all results agree perfectly. Then, up to about $t=55 \mathrm{\mu s}$, the droplet height is only slightly underestimated with ${ }_{530}$ AIFM.

\subsubsection{Experiment 4}

In our fourth experiment, we simulate the impact of the same droplet as in Exp. 3 on another substrate, i.e. on S107 with equilibrium contact angle $107^{\circ}$, which corresponds to Exp. 4 in Table 3.

The droplets' shapes are given in Figure 8(a). Up to about $t=5 \mu \mathrm{s}$, the droplet behaves similarly to Exp. 3. Thus, we see the development of two layers from $t=2 \mu$ s onwards which are slightly stretched throughout the spreading phase, and the rim of the droplet remains its highest part. Then, due to the higher contact angle, the droplet recedes much further at $t=10 \mu \mathrm{s} \quad 540$ and is about to rebound at $t=50 \mu \mathrm{s}$.

In Figure 8(b), we compare the results of the practical experiment (first row) with simulation results by AIFM (second row) and by CCA (third row). Up to about $t=4 \mu \mathrm{s}$, the droplet shapes in both simulations are quite close to those observed in the practical experiment. As previously, the diameter of the droplet is overestimated with CCA. In addition, at $t=5 \mu \mathrm{s}$, the droplet is more curved than the one computed by AIFM and found from the practical experiment. Furthermore, this droplet recoils much faster and has already hit the top of the simulation wall at $t=50 \mu \mathrm{s}$. In contrast to this approach, the droplet shapes computed by AIFM are very close to the experimental 550 snapshots at all times.

All of these findings are confirmed by the computation of the dimensionless diameter $D^{*}=d / d_{0}$ over time in Figure 8(c). Remarkably, the diameter 


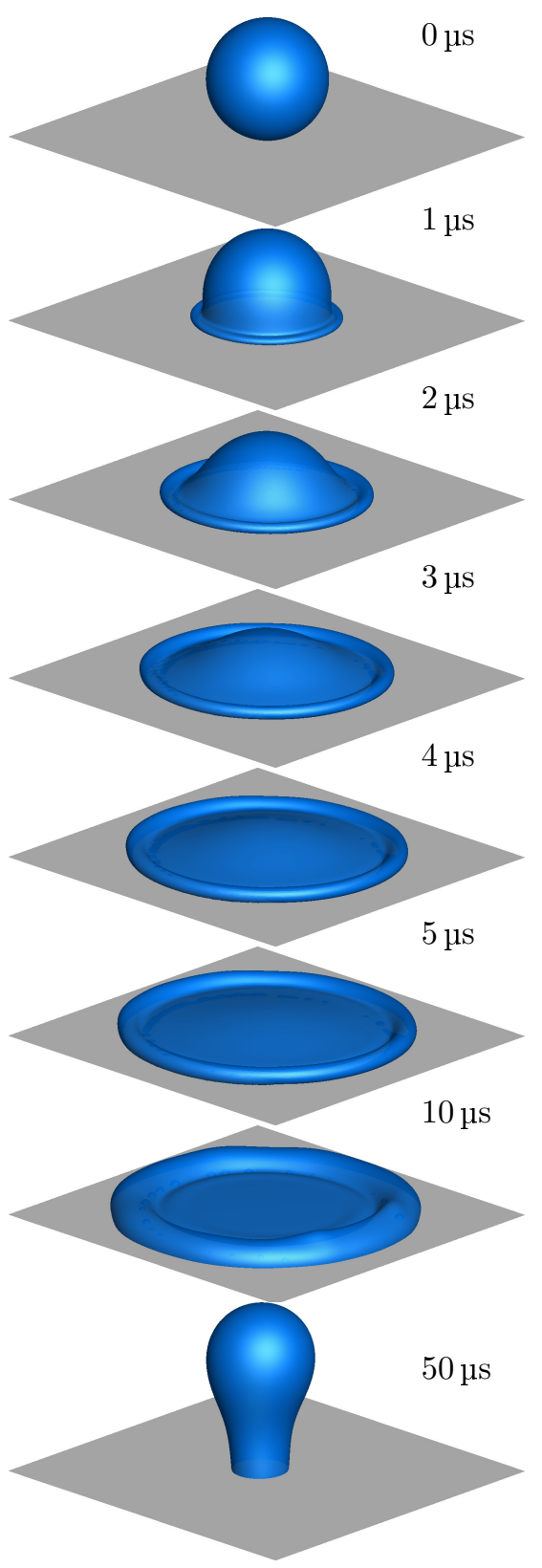

(a) Droplet shapes computed by AIFM for Exp. 4.0 from Table 3.

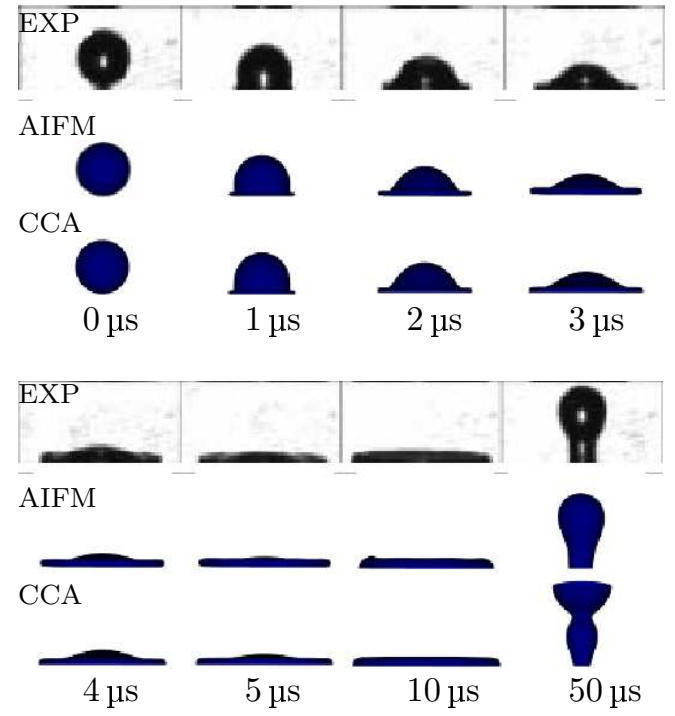

(b) Comparison of the practical experiment (first row), Exp. 4.0 (second row) and Exp. 4.1 (third row); cf. Table 3.

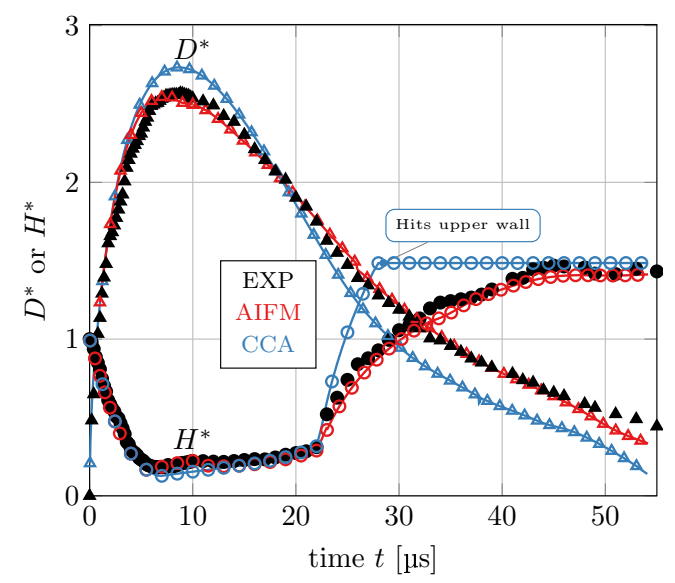

(c) Dimensionless droplet diameter $D^{*}$ (triangles) and height $H^{*}$ (circles) vs. time: EXP (black), AIFM (red) and CCA (blue).

Figure 8: Distilled water impacting on S107 with equilibrium contact angle $107^{\circ}$, impact speed $12.2 \mathrm{~m} \mathrm{~s}^{-1}$, and droplet size $50.5 \mu \mathrm{m}$. Experimental data and figures used with permission by H. Dong [3]. 
computed by AIFM and the practical experiment agree almost perfectly at all times. In contrast, the diameter computed by CCA deviates increasingly 555 from $t=2 \mu \mathrm{s}$ onwards and the maximum droplet diameter is vastly overestimated. Then, from about $20 \mu \mathrm{s}$ onwards, the diameter is underestimated which indicates the much earlier rebound of this droplet.

In the same Figure 8(c), the dimensionless droplet height $H^{*}=h / d_{0}$ is plotted over time. Again, we observe an amazing agreement between the 560 droplet height computed by AIFM and obtained in the practical experiment. From $0 \mu \mathrm{s}$ to $20 \mu \mathrm{s}$ the results of CCA are also in perfect agreement with the experiment. Then, due to the faster recoil of the droplet, the droplet height increases much more dramatically until the droplet hits the upper wall of the simulation domain.

\subsection{Comparison to Lattice Boltzmann simulations}

In this subsection, we compare our results to those in [4]. Here, the same numerical experiments are conducted with a Lattice Boltzmann simulation (LBS), and the contact angle is reconstructed by the empirical 'Hoffman's function' and the capillary number. Note that the final simulation time for us is $55 \mu \mathrm{s}$ and $65 \mu \mathrm{s}$ for LBS. Both simulation approaches show equally good results with differences varying for the different experiments.

For Exp. 1 both the droplet height and width are captured in a very similar manner. Especially, the final droplet diameter corresponds to the analytic prediction

$$
D^{*}=\left(\frac{8}{\tan \left(\frac{\theta_{e}}{2}\right)\left(3+\tan ^{2}\left(\frac{\theta_{e}}{2}\right)\right)}\right)^{\frac{1}{3}}
$$

which assumes volume conservation and a spherical cap. For Exp. 2 LBS is closer to the experiment than AIFM and predicts the final diameter according to (19) very well. However, LBS underestimates the minimum height of the 575 droplet, which is captured very well with AIFM. For Exp. 3, we have equally good results for AIFM and LBS. Then, in Exp. 4, AIFM is very close to the physical experiment and fares much better than LBS. Again, we capture the maximum droplet width and minimum height almost perfectly which is not possible with LBS. Both simulations predict the droplet width at $55 \mu$ s well, 580 but only AIFM also gives a very good estimation of the droplet height.

To summarize, both simulation methods are able to predict results that are very close to the experiment. The LBS provides better results for lower 
contact angles while the quality of AIFM seems to increase for the larger ${ }_{585}$ Weber number. In all experiments, when the droplet recedes for the first time after impact, the maximum droplet height is underestimated by LBS but captured very well by AIFM.

\subsection{Irregularities at the interface}

After the discussion on the individual experiments, we would like to comment on some of the irregularities in the interfaces visible in some parts of Figures 7-8. In these figures, the symmetry of the droplets is partly lost after spreading. These irregularities could not be traced back to a single cause. We assume that under-resolved structures, accumulated interface reconstruction errors in the CLSVOF method, as well as the need of time step adaption for the contact angle boundary condition all contribute to the problem; see [20] for details. Further research will certainly shed light on these difficulties and, in a first step, all of these irregularities should improve with finer mesh sizes and finer time steps. Additionally, the CLSVOF method can certainly benefit from a 'smoother-in-time' implementation of the contact angle: For the level-set method, the contact angle is part of the boundary condition for the reinitialization equation, which takes several artificial time-steps to reach its equilibrium value, similar to the approach in [22]. A further option is the method by Spelt [32] where contact line position and contact angle are determined iteratively. Last, our conversion from meters to millimeters for all input parameters can also have an adverse effect on the accuracy of our computations. Although the LS function remains well-defined due to the conversion, the liquid density and gas viscosity become smaller than $\mathcal{O}\left(10^{-8}\right)$ which might lead to accuracy issues within our flow solver.

\subsection{Remarks}

610

All in all, our results with AIFM showed very good agreement with the practical experiments concerning the droplet shapes, droplet diameter and its height. As expected, AIFM displayed considerable improvement as opposed to CCA for all numerical experiments. In particular, we anticipated good results by both contact angle approaches at early times after impact, when inertial forces are dominant, which could be confirmed by the experiments. However, the droplet diameter tends to be overestimated with CCA.

Since the Weber number for Exp. 3 and 4 is considerably larger, we observed an exceedingly good agreement of AIFM and the practical experiments. Here, the difference between AIFM and CCA was less than for Exp. 1 and 2, 
where surface tensions forces were dominant. In all four experiments, the ${ }_{620}$ diameter/height curve computed by AIFM showed a similar development as the one in the practical experiment.

\section{Conclusion}

In this article, we coupled an asymptotic version of Shikhmurzaev's interface formation model (AIFM) with our three-dimensional incompressible two-phase Navier-Stokes solver NaSt3DGPF. Along with the CLSVOF method for the treatment of the free surface, this combination yielded a highly effective tool for the numerical simulation of micron-scale droplet impact in three spatial dimensions. For different impact speeds and wetting characteristics, AIFM yielded impressive results compared with practical experiments. Both the droplet diameter and the droplet height were very close to that from experimental measurements and AIFM clearly outperformed the constant angle approach (CCA), where the dynamic contact angle was fixed to its equilibrium value for the whole simulation.

So far, our results are already a significant improvement over other ap- $\quad 635$ proaches tackling the moving contact line problem. Our approach could be further improved by the computation of the contact angle in every grid cell along the contact line. For our simulations of droplet impact, the assumption of axisymmetric spreading is reasonable. However, aiming at simulations of further wetting processes such as curtain coating, AIFM has to be applied ${ }_{640}$ pointwise at the whole contact line.

All in all, the interface formation model in its asymptotic formulation in conjunction with the CLSVOF method for the treatment of the free interface confirmed to be a high quality and easily adaptable tool for the computation of dynamic contact angles for droplet impact simulations.

\section{Acknowledgments}

We would like to express our sincere gratitude to Dr. Hongming Dong for providing experimental results of the droplet impacts and the permission to use the corresponding pictures in this article.

This research did not receive any specific grant from funding agencies in 650 the public, commercial, or not-for-profit sectors. 


\section{References}

[1] Y. Shikhmurzaev, Capillary flows with forming interfaces, Chapman \& Hall/CRC, 2008.

[2] D. Bolleddula, Droplet impact and spreading of viscous dispersions and volatile solutions, Ph.D. thesis, University of Washington (2011).

[3] H. Dong, W. Carr, D. Bucknall, J. Morris, Temporally-resolved inkjet drop impaction on surfaces, AIChE Journal 53 (10) (2007) 2606-2617.

[4] T. Lee, L. Liu, Lattice Boltzmann simulations of micron-scale drop impact on dry surfaces, Journal of Computational Physics 229 (20) (2010) 8045-8063.

[5] J. Billingham, On a model for the motion of a contact line on a smooth solid surface, European Journal of Applied Mathematics 17 (3) (2006) $347-382$.

[6] D. Bothe, J. Prüss, On the interface formation model for dynamic triple lines, in: Mathematical Fluid Dynamics, Present and Future, Springer, 2016, pp. 25-47.

[7] J. Sprittles, Y. Shikhmurzaev, Finite element simulation of dynamic wetting flows as an interface formation process, Journal of Computational Physics 233 (2013) 34-65.

[8] J. Sprittles, Y. Shikhmurzaev, The dynamics of liquid drops and their interaction with solids of varying wettabilities, Physics of Fluids 24 (8).

[9] J. Sprittles, Y. Shikhmurzaev, Coalescence of liquid drops: Different models versus experiment, Physics of Fluids 24 (12).

[10] S. Decent, Hydrodynamic assist and the dynamic contact angle in the coalescence of liquid drops, IMA Journal of Applied Mathematics 71 (5) (2006) 740-767.

[11] J. Monnier, P. Witomski, Analysis of a local hydrodynamic model with Marangoni effect, Journal of Scientific Computing 21 (3) (2004) 369-403. 
[12] D. Sibley, N. Savva, S. Kalliadasis, Slip or not slip? A methodical ${ }_{680}$ examination of the interface formation model using two-dimensional droplet spreading on a horizontal planar substrate as a prototype system, Physics of Fluids 24 (8).

[13] J. Göhl, A. Mark, S. Sasic, F. Edelvik, An immersed boundary based dynamic contact angle framework for handling complex surfaces of mixed 685 wettabilities, International Journal of Multiphase Flow 109 (2018) 164177.

[14] M. Griebel, T. Dornseifer, T. Neunhoeffer, Numerical simulation in fluid dynamics: A practical introduction, Vol. 3, SIAM, Philadelphia, 1998.

[15] NaSt3DGPF, A parallel 3d free surface flow solver, http://wissrech. $\quad 690$ iam.uni-bonn.de/research/projects/NaSt3DGPF/.

[16] S. Osher, J. A. Sethian, Fronts propagating with curvature-dependent speed: Algorithms based on Hamilton-Jacobi formulations, Journal of Computational Physics 79 (1) (1988) 12-49.

[17] J. Brackbill, D. Kothe, C. Zemach, A continuum method for modeling ${ }^{695}$ surface tension, Journal of Computational Physics 100 (1992) 335-354.

[18] R. Croce, M. Griebel, M. Schweitzer, Numerical simulation of bubble and droplet deformation by a level set approach with surface tension in three dimensions, International Journal for Numerical Methods in Fluids 62 (9) (2010) 963-993.

[19] S. Groß, Numerical methods for three-dimensional incompressible twophase flow problems, Dissertation, IGPM, RWTH Aachen (2008).

[20] M. Klitz, Numerical simulation of droplets with dynamic contact angle, Dissertation, Institute for Numerical Simulation, Bonn University (2014).

[21] M. Griebel, M. Klitz, CLSVOF as a fast and mass-conserving extension of the level-set method for the simulation of two-phase flow problems, Numerical Heat Transfer, Part B: Fundamentals 71 (1) (2017) 1-36.

[22] M. Sussman, An adaptive mesh algorithm for free surface flows in general geometries, in: A. Vande Wouwer, P. Saucez, W. E. Schiesser (Eds.), 710 Adaptive Method of Lines, Chapman \& Hall/CRC, 2001, pp. 207-231. 
[23] M. Griebel, M. Klitz, Simulation of droplet impact with dynamic contact angle boundary conditions, in: Singular Phenomena and Scaling in Mathematical Models, Springer International Publishing Switzerland, 2013, pp. 297-325.

[24] H. Moffatt, Viscous and resistive eddies near a sharp corner, Journal of Fluid Mechanics 18 (1) (1964) 1-18.

[25] R. Croce, M. Griebel, M. Schweitzer, A parallel level-set approach for two-phase flow problems with surface tension in three space dimensions, Preprint 157, Sonderforschungsbereich 611, Universität Bonn (2004).

[26] INSGrid, High-Performance Cluster Computers at the INS and SFB 1060, http://wissrech.ins.uni-bonn.de/research/atacama/ (2013).

[27] ParaView, Paraview user's guide (v3.10), http://www.paraview.org/ (2011).

[28] Tecplot, Tecplot 360 users manual, http://www.tecplot.com/ (2013).

[29] W. Lorensen, H. Cline, Marching cubes: A high resolution 3d surface construction algorithm, in: ACM Siggraph Computer Graphics, Vol. 21, ACM, 1987, pp. 163-169.

[30] P. Zaspel, M. Griebel, Photorealistic visualization and fluid animation: Coupling of Maya with a two-phase Navier-Stokes fluid solver, Computing and Visualization in Science 14 (8) (2011) 371-383.

[31] T. Blake, Y. Shikhmurzaev, Dynamic wetting by liquids of different viscosity, Journal of Colloid and Interface Science 253 (1) (2002) 196202.

[32] P. Spelt, A level-set approach for simulations of flows with multiple moving contact lines with hysteresis, Journal of Computational Physics 207 (2) (2005) 389-404. 